Research Paper

\title{
Increase in Peripheral Blood Intermediate Monocytes is Associated with the Development of Recent-Onset Type 1 Diabetes Mellitus in Children
}

\author{
Xiaoya Ren ${ }^{1,2,3^{*}}$, Wenjun Mou ${ }^{1,3 *}$, Chang Su${ }^{1,2}$, Xi Chen ${ }^{1,3}$, Hui Zhang ${ }^{1,3}$, Bingyan Cao ${ }^{1,2}$, Xiaoqiao Li1,2, Di \\ $\mathrm{Wu}^{1,2}$, Xin $\mathrm{Ni}^{1,4}$, Jingang Gui1,3凶${ }^{\square}$, Chunxiu Gong1,2凶 \\ 1. Key Laboratory of Major Diseases in Children by Ministry of Education, Beijing Children's Hospital, Capital Medical University, Beijing, China. \\ 2. Department of Endocrinology, Genetics and Metabolism, Beijing Children's Hospital, Capital Medical University, Beijing, China. \\ 3. Laboratory of Immunology, Beijing Pediatric Research Institute, Beijing Children's Hospital, Capital Medical University, Beijing, China. \\ 4. Department of Otolaryngology, Head and Neck Surgery, Beijing Children's Hospital, Capital Medical University, Beijing 100045, P.R. China. \\ *These authors contributed equally to this study.
}

$\square$ Corresponding authors: Dr. Jingang Gui, Laboratory of Immunology, Beijing Pediatric Research Institute, Beijing Children's Hospital, Capital Medical University, Beijing, 100045, China. Email: guijingang@bch.com.cn and Dr. Chunxiu Gong, Department of Endocrinology, Genetics and Metabolism, Beijing Children's Hospital, Capital Medical University, Beijing, 100045, China. Email: gongchunxiu@bch.com.cn.

(1) Ivyspring International Publisher. This is an open access article distributed under the terms of the Creative Commons Attribution (CC BY-NC) license (https://creativecommons.org/licenses/by-nc/4.0/). See http://ivyspring.com/terms for full terms and conditions.

Received: 2016.03.27; Accepted: 2016.12.05; Published: 2017.02.05

\begin{abstract}
Monocytes play important roles in antigen presentation and cytokine production to achieve a proper immune response, and are therefore largely implicated in the development and progression of autoimmune diseases. The aim of this study was to analyze the change in the intermediate $(\mathrm{CD} 14+\mathrm{CD} 16+)$ monocyte subset in children with recent-onset type 1 diabetes mellitus (TIDM) and its possible association with clinical parameters reflecting islet $\beta$-cell dysfunction. Compared with age- and sex-matched healthy controls, intermediate monocytes were expanded in children with TIDM, which was positively associated with hemoglobin AIC and negatively associated with serum insulin and C-peptide. Interestingly, the intermediate monocytes in TIDM patients expressed higher levels of human leukocyte antigen-DR and CD86, suggesting better antigen presentation capability. Further analysis revealed that the frequency of CD45RO+CD4+ memory $T$ cells was increased in the TIDM patients, and the memory $T$ cell content was well correlated with the increase in intermediate monocytes. These results suggest that expanded intermediate monocytes are a predictive factor for the poor residual islet $\beta$-cell function in children with recent-onset TIDM.
\end{abstract}

Key words: Children; Type 1 Diabetes Mellitus; Intermediate Monocytes; Islet Beta Cell Function; Memory T cells.

\section{Introduction}

Type 1 diabetes mellitus (T1DM) is an autoimmune disease in which the function of insulin-secreting pancreatic $\beta$-cells is impaired due to autoreactive immune cell-mediated destruction (insulitis). T1DM is characterized by dysregulation of blood glucose caused by $\beta$-cell insufficiency accompanied by increased hemoglobin $\mathrm{A} 1 \mathrm{C}(\mathrm{HbA} 1 \mathrm{c})$ $[1,2]$. Several studies suggest that the development of T1DM is strongly associated with different immune cell subsets, including monocytes [3-5]. Specifically, an increase in the monocyte population has been shown to trigger $\beta$-cell destruction during insulitis [6].

Human peripheral monocytes act as antigen-presenting cells (APCs) to activate $\mathrm{T}$ cells during inflammatory conditions $[7,8]$, and to secrete cytokines shaping T-cell differentiation [9]. Monocytes are comprised of heterogeneous subgroups that can be classified as classical 
(CD14++CD16-, 85\%), intermediate (CD14+CD16+, about 5\%), and non-classical (CD14dimCD16++, about $10 \%$ ) based on the differential expression levels of CD14 (a lipopolysaccharide [LPS] receptor) and CD16 (Fc $\gamma$ RIII) [10-12]. Increasing evidence has shown that intermediate monocytes exert an antigen-presenting function with a dendritic cell-like feature [13]. It has been documented that intermediate monocytes exhibit enriched expression in antigen-presenting-related factors such as major histocompatibility complex class II (MHC-II) subunits, CD74 (class II invariant chain), human leukocyte antigen (HLA)-DO, as well as CD40 [14]. Moreover, intermediate monocytes mediate inflammation and the pathogenesis of infections [15].

Intermediate monocytes have been shown to expand in many inflammatory and autoimmune conditions, including chronic kidney disease, active rheumatoid arthritis, coronary artery disease, and type 2 diabetes [16-19]. Significantly increased intermediate monocytes in patients with juvenile-onset T1DM were demonstrated to produce more tumor necrosis factor-alpha (TNF- $\alpha$ ), an effective inflammatory factor [6, 10]. In fact, upon antigen stimulation, intermediate monocytes become the main producers of inflammatory factors, including interleukin (IL)-1 $\alpha$, IL-6, and TNF- $\alpha[20]$, and TNF- $\alpha$ has been shown to correlate with the severity of T1DM [6, 21-23].

The monocytes of diabetic patients are able to induce $\mathrm{CD} 4+\mathrm{T}$ cells to produce proinflammatory cytokines such as interferon-gamma (IFN- $\gamma$ ), TNF- $\alpha$, and IL-17 [24]. Monocytes were also found to promote the expansion of memory $\mathrm{T}$ cells in patients with human immunodeficiency virus (HIV) [25]. Respiratory monocytes enhance the responses of CD4+ memory $\mathrm{T}$ cells to mucosal immunization with recombinant adenovirus-based vaccines [26]. However, the action of the intermediate monocytes in children with recent-onset T1DM, and the consequences for memory $\mathrm{T}$ cell responses have not been fully investigated.

Here, we aimed to investigate the changes and functions of intermediate monocytes and to analyze the possible association of the change in the intermediate monocyte subset with clinical parameters reflecting islet $\beta$-cell insufficiency in children with recent-onset T1DM. The increase in this subset demonstrated a good correlation with worse residual islet $\beta$-cells function. We also found that children with recent-onset T1DM that had a higher intermediate monocyte population had a greater number of memory T cells, which secreted high levels of IL-2 and IFN- $\gamma$. Collectively, these results suggest that expansion of intermediate monocytes is a poor prognostic factor for the progress of T1DM in children.

\section{Material and methods}

\section{Subjects}

A total of 54 patients ( 30 boys, 24 girls; mean age $7.3 \pm 3.9$ years) with recent-onset T1DM were enrolled in this study between January 2015 and July 2016 from Beijing Children's Hospital. Patients with micro-vascular complications, and coexisting autoimmune, chronic, and acute inflammatory diseases were excluded from the study. The clinical characteristics of the patients are presented in Table 1. Forty-nine age- and sex-matched healthy children (26 boys, 23 girls; mean age $6.1 \pm 3.9$ years) were included as a healthy control (HC) group. Thirty-nine age- and sex-matched patients with Graves' disease (19 boys, 20 girls; mean age $7.6 \pm 3.6$ years) were included as a non-T1DM autoimmune control group. The study was approved by the ethics committee of Beijing Children's Hospital, which acts in compliance with ethical standards defined by the Declaration of Helsinki. Written consent for research purposes was provided by all participants and their parents or legal guardians.

Table 1. Clinical characteristic of patients with type 1 diabetes.

\begin{tabular}{lll}
\hline Clinical parameter & $\begin{array}{l}\text { Mean } \pm \text { standard } \\
\text { deviation }\end{array}$ & $\begin{array}{l}\text { range of reference } \\
\text { value }\end{array}$ \\
\hline Age (years) & $7.3 \pm 3.9$ & \\
Duration of diabetes (days) & $19.5 \pm 9.1$ & \\
HbA1c $(\%)$ & $11.8 \pm 2.5$ & $4.0-6.0$ \\
Insulin $(\mathrm{IU} / \mathrm{ml})$ & $3.2 \pm 2.3$ & $6.0-27.0$ \\
C-peptide $(\mathrm{ng} / \mathrm{ml})$ & $0.9 \pm 0.7$ & $1.1-5.0$ \\
\hline
\end{tabular}

\section{Sample collection}

Serum and peripheral blood mononuclear cells (PBMCs) were isolated from ethylenediaminetetraacetic acid-treated blood samples collected from all participants. The PBMCs were separated by standard Ficoll-Hypaque density centrifugation at $1000 \mathrm{rpm}$ for $20 \mathrm{~min}$.

\section{Blood measurements}

The serum HbA1c level was determined by high-performance liquid chromatography using the Ark-8150 system (Arkray Inc. Kyoto, Japan). Fasting insulin and C-peptide levels were analyzed via immune chemiluminometric assays (Roche cobas E601 analyzer, Roche Diagnostics, USA). The intraand inter-assay coefficients of variation were $<5 \%$ and $<10 \%$, respectively.

\section{Surface phenotypic marker expression}

The absolute counts of total lymphocytes, monocytes, and neutrophils in the peripheral blood of 
all subjects were measured by flow cytometry with a Guava easyCyte 8 system (Merck Millipore, USA). Cell-surface monocyte phenotypic analysis was performed after staining with human anti-CD14 [1] and anti-CD16 [1]. Fluorochrome-conjugated monoclonal antibodies allophycocyanin (APC)-CD40, phycoerythrin (PE)-CD86, APC-HLA-DR, PE-CD11b, APC-CD11C, APC-CD62L, PE-CD68, PE-CD4, peridinin chlorophyll protein complex (PerCP)-CD3, PE-CD45RO, APC-CCR7, fluorescein isothiocyanate (FITC)-CD40L, and PE-CD163 were purchased from BD Biosciences Pharmingen (Franklin Lanes, NJ, USA).

\section{Intracellular staining}

Isolated monocytes $\left(10^{5}\right)$ and PBMCs were incubated with $100 \mathrm{ng} / \mathrm{ml}$ LPS (Sigma Aldrich), 5 ng/ml IL-2 (Cell Signaling Technologies), $50 \mathrm{ng} / \mathrm{ml}$ phorbol-12-myristate-13-acetate (PMA, Merck), and 1 $\mu \mathrm{g} / \mathrm{ml}$ ionomycin (Sigma Aldrich) for $37^{\circ} \mathrm{C}, 5 \mathrm{~h}$, respectively; and GolgiStop (BD Biosciences) was added for the final $3 \mathrm{~h}$. Monocytes were stained with FITC-CD14 and PerCP-CD16 (Biolegend), and PBMCs were stained with FITC-CD45RO, PE-CD4, PerCP-CD3, and APC-CCR7 for $30 \mathrm{~min}$. Cells were fixed and permeabilized using the protocol from the Cytofix/Cytoperm kit (BD Biosciences). Then, the monocytes were stained with anti-TNF- $\alpha$ (Biolegend) and anti-IL-6 (Biolegend), and the PBMCs were stained with anti-IFN- $\gamma$ (BD Biosciences) and anti-IL-2 (BD Biosciences) for $30 \mathrm{~min}$.

Expression of cell-surface markers was assessed using flow cytometry (FACSCalibur, BD, USA) after gating of live cells, and analyzed according to scatter characteristics using FlowJo software.

\section{Statistical analysis}

Pairwise comparisons of mean lymphocyte and monocyte cell counts among the HC, Graves, and diabetic groups were performed with the Student's $t$-test. Spearman's correlations were used to compare the associations among intermediate monocyte subsets, clinical parameters, and memory $\mathrm{T}$ cells in the T1DM group. The level of significance was set at $p<$ 0.05 . Significant differences were calculated with GraphPad Prism 4.0 using an unpaired two-tailed t-test.

Table 2. Absolute counting of different types of cells.

\begin{tabular}{lllllll}
\hline $\begin{array}{l}\text { Absolute number } \\
(\text { cells } / \mathrm{mL})\end{array}$ & $\begin{array}{l}\text { Lymphocytes } \\
\left(\times 10^{6}\right)\end{array}$ & $\begin{array}{l}\text { Monocytes } \\
\left(\times 10^{5}\right)\end{array}$ & $\begin{array}{l}\text { Neutrophils } \\
\left(\times 10^{6}\right)\end{array}$ & $\begin{array}{l}\text { Memory T cells } \\
\left(\times 10^{5}\right)\end{array}$ & $\begin{array}{l}\text { CD14++CD16- } \\
\left(\times 10^{5}\right)\end{array}$ & $\begin{array}{l}\text { CD14+CD16+ } \\
\left(\times 10^{4}\right)\end{array}$ \\
\hline HC & $1.43 \pm 0.78$ & $2.10 \pm 1.20$ & $1.14 \pm 0.93$ & $2.64 \pm 2.00$ & $1.27 \pm 0.79$ & $2.38 \pm 1.51$ \\
Graves & $1.58 \pm 1.02$ & $1.57 \pm 0.97$ & $1.73 \pm 1.60$ & $2.90 \pm 2.40$ & $0.69 \pm 0.41$ & $0.74 \pm 0.52$ \\
T1DM & $1.77 \pm 0.83$ & $2.91 \pm 1.77$ & $1.38 \pm 0.82$ & $4.88 \pm 2.97$ & $1.40 \pm 1.03$ & $0.57 \pm 0.45$ \\
\hline
\end{tabular}

\section{Results}

\section{The increase of intermediate monocytes is specific to TIDM}

The intermediate monocytes have been shown to contribute to the pathogenesis of pediatric T1DM, a typical autoimmune and inflammatory disease $[6,24]$. In the present study, we sought to determine the phenotype and function of intermediate monocytes in children with recent-onset T1DM.

Our results showed that the percentage of total monocytes was increased in the children with T1DM compared with the age- and sex-matched HC and Graves' disease groups (Fig. 1A). The absolute count of monocytes in the children with T1DM was significantly higher than that in the HC group and Graves patients. However, there were no differences in the populations of lymphocytes and neutrophils among the T1DM, Graves, and HC groups. The absolute counts of total lymphocytes and neutrophils in the peripheral blood of T1DM patients showed no differences from those of the HC group.

When monocyte subsets were further examined, the proportion of each subset relative to total monocytes was also found to vary in the T1DM group (Fig. 1B). Compared with the age- and sex-matched HC group and patients with Graves, the ratio of intermediate monocytes was specifically increased in the T1DM children (Fig. 1C). Absolute cell counting also revealed a specific increase in the intermediate monocytes of children with T1DM (Table 2).

\section{Relationship between intermediate monocytes and the remnant islet $\beta$-cell function in TIDM children}

A decrease in serum insulin concentration and C-peptide is a typical pathophysiological change recognized in T1DM patients, and has been commonly used for evaluating the remnant islet $\beta$-cell function. $\mathrm{HbA1c}$ is a recognized standard clinical marker of glycemic control, and is significantly increased in T1DM. These clinical indices are therefore currently used to assess the condition of T1DM patients. Recent studies have also shown that the expansion of intermediate monocytes could trigger the destruction process of $\beta$-cell $[6,10]$. Therefore, it is important to determine the relationship between the intermediate monocytes and clinical indices attributed to pancreas islet functions. 
A
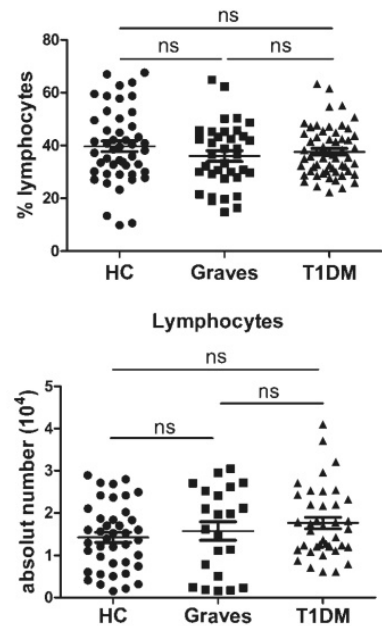

B

$\mathrm{HC}$

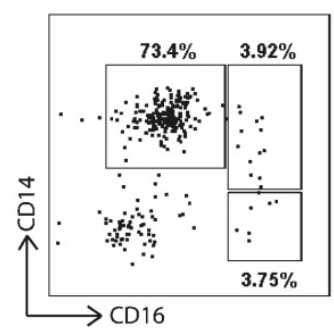

C
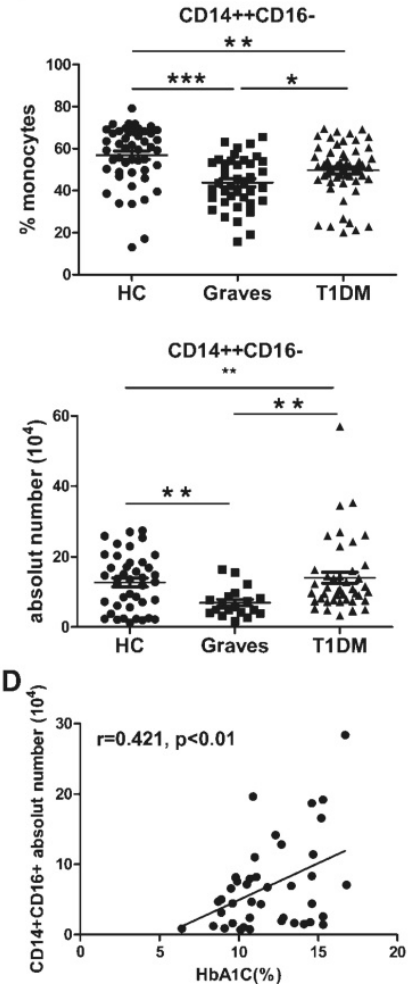
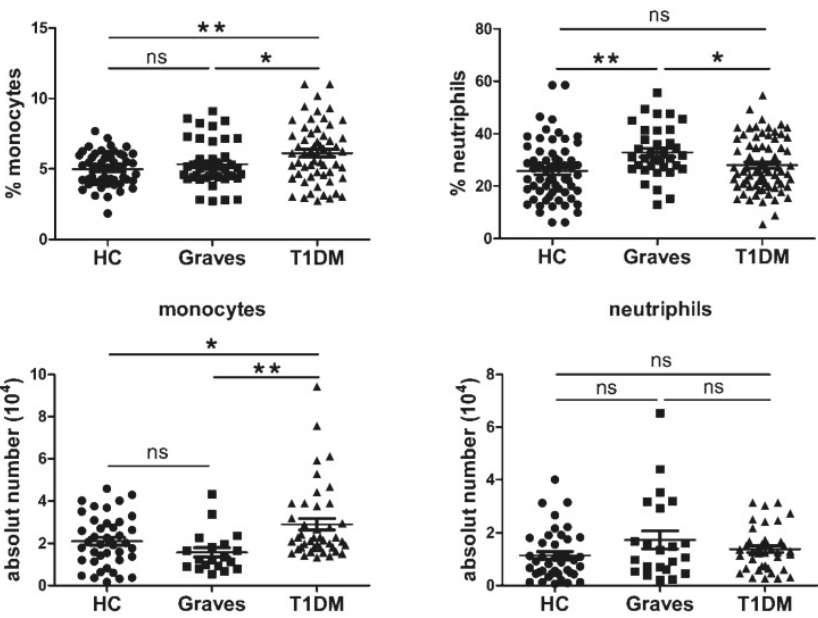

Graves

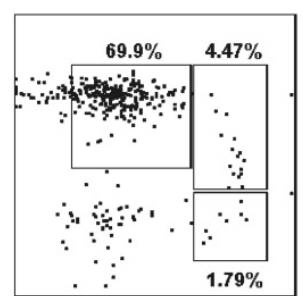

T1DM

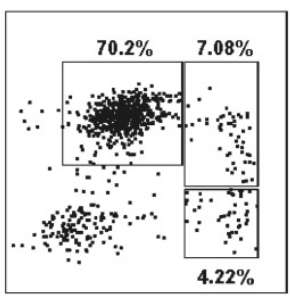

CD14dimCD16++

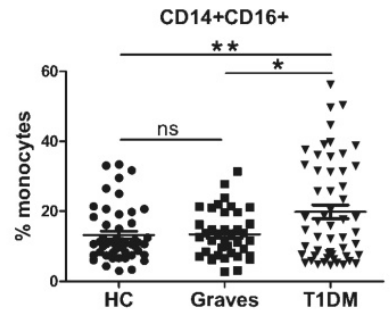

CD14+CD16+
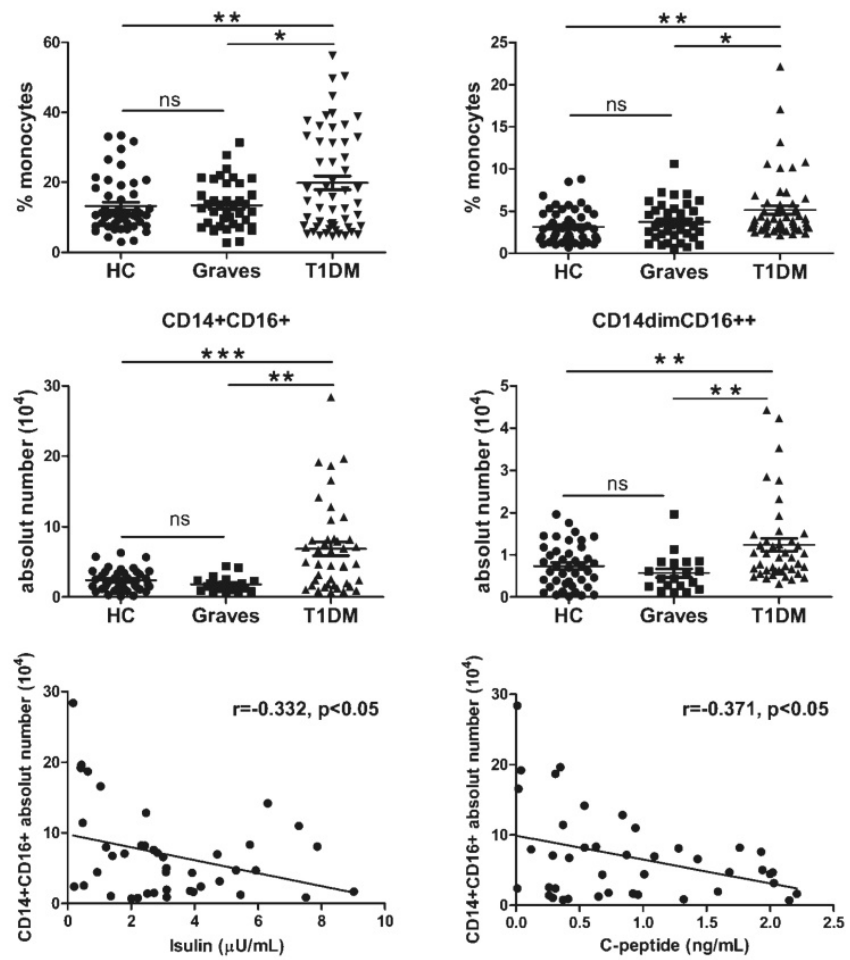

Figure 1. (A) The percentages of different cell types in the blood. (A) Left panel: the proportion (\%) of total lymphocytes. Middle panel: the proportion (\%) of total monocytes. Right panel: the proportion (\%) of total neutrophils. (B) The whole blood samples were stained with antibodies against CDI4 and CD16 molecules to identify the monocyte subsets as follows: CD14++CD16- (classical monocytes), CD14+CD16+ (intermediate monocytes), and CDI4dimCD16++ (non-classical monocytes). (C) The percentages of the three monocyte subsets among blood monocytes. Left panel: the proportion (\%) of CDI4++CD16- cells. Middle panel: the proportion (\%) of CD14+CD16+ cells. Right panel: the proportion (\%) of CD14dimCD16++ cells. (D) Left panel: correlation of the HbAlc level with the absolute number of CD14+CD16+ monocytes $(r=0.421, p<0.01)$. Middle panel: correlation of the insulin level with the absolute number of CDI4+CD16+ monocytes $(r$ $=-0.332, p<0.05)$. Right panel: correlation of the $C$-peptide level with the absolute number of CD14+CD16+ monocytes $(r=-0.371, p<0.05)$. Each dot represents a different individual; ns, $\mathrm{p}>0.05 ; *, \mathrm{p}<0.05 ; * *, \mathrm{p}<0.01$; ***, $\mathrm{p}<0.001$. 
All of the T1DM patients recruited in this study showed a dramatic increase in HbA1c $(11.8 \% \pm 2.5$, reference value $4.0-6.0 \%$ ), and a significant decrease in both the serum insulin concentration $(3.2 \pm 2.3$ $\mathrm{IU} / \mathrm{ml}$, reference value $6.0-27 \mathrm{IU} / \mathrm{ml}$ ) and serum C-peptide level $(0.9 \pm 0.7 \mathrm{ng} / \mathrm{ml}$, reference value $1.1-5.0 \mathrm{ng} / \mathrm{ml}$ ) (Table 1). Correlation analysis revealed that T1DM patients with higher HbA1c levels had greater numbers of peripheral blood intermediate monocytes (Fig. 1D, left panel). Moreover, an inverse correlation was observed between the absolute number of intermediate monocytes and the concentration of insulin and C-peptide, respectively, in T1DM children (Fig. 1D, middle and right panels). We also analyzed the correlation in $\mathrm{HC}$, and found there is no correlation between intermediate and the islet $\beta$-cell function (Supplementary Figure 2A, 2B, $2 \mathrm{C})$. The correlation between intermediate and the remnant islet $\beta$-cell function is specific in T1DM. These results imply that the enrichment of intermediate monocytes in T1DM has a destructive effect on the function of residual islet $\beta$-cells. Therefore, we next examined the phenotype and function of APCs in the intermediate monocyte subset in children with T1DM.

\section{Phenotype of the intermediate monocytes in children with TIDM}

To further characterize the expression of cell-surface markers related to inflammatory chemotaxis, and activation and phagocytosis of the CD14+CD16+ monocyte subset, we investigated the expression of CD11b, CD11c, CD68, CD62L, and
CD163 monocytes from the T1DM patients, Graves patients, and HC group.

The integrin Mac-1 (CD11b) is one of the most well-studied leukocyte adhesion molecules mediating leukocyte adhesion and migration to regulate the inflammatory response [27]. We found that the intermediate monocyte population from the T1DM patients showed higher CD11b expression compared to those of the Graves and HC groups (Fig. 2A). $\mathrm{CD} 62 \mathrm{~L}$ is an adhesion molecule that is present on the surface of inflammatory monocytes, and its expression has been linked to the capacity of monocytes to preferentially migrate to the sites of inflammation [28]. The percentage of CD62L on intermediate monocytes was significantly higher in the T1DM group (Fig. 2C). Overall, these data showed the enhancement of monocyte inflammatory chemotaxis in children with T1DM.

To determine the changes in the activation of intermediate monocytes, we analyzed CD11c, a marker of activated monocytes/macrophages [27]. As expected, the expression of CD11c was significantly higher in the T1DM patients compared with that in the Graves and HC group, respectively (Fig. 2B).

To investigate possible differences in the phagocytosis of intermediate monocytes, we analyzed the expression of CD68 and CD163, a marker of tissue macrophages and a member of the scavenger receptor cysteine-rich family of proteins, respectively [29]. Specifically, intermediate monocytes from the T1DM patients showed higher surface levels of CD68 and CD163 than those derived from the Graves patients or HC group (Fig. 2D and E).
A

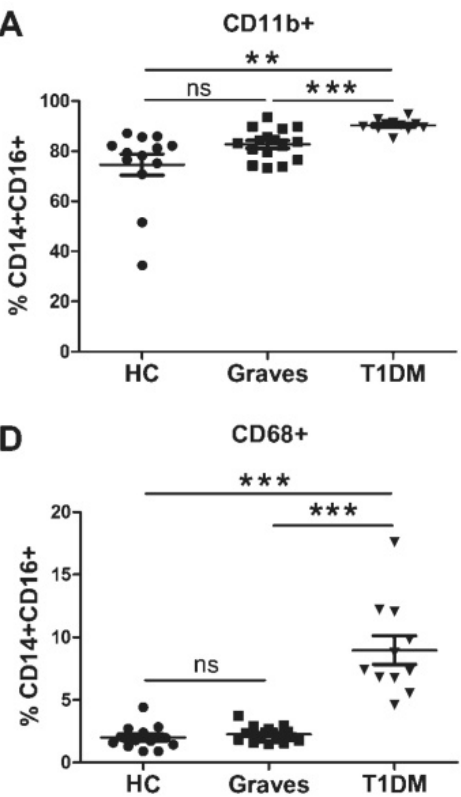

B

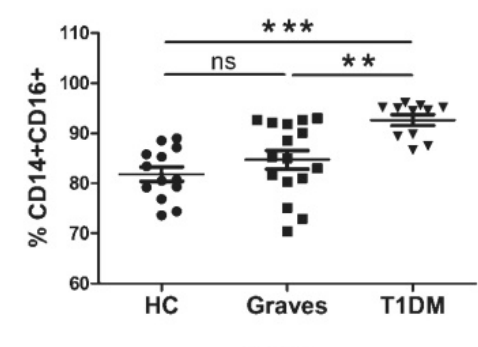

E

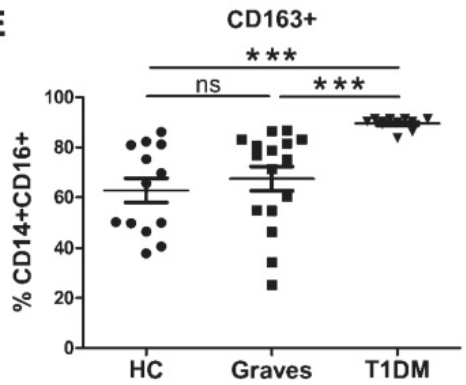

C CD62L+

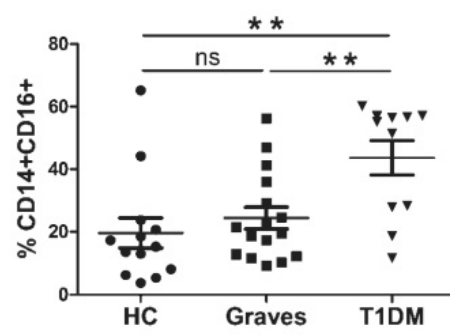

Figure 2. The phenotype of $C D 14+C D 16+$ monocytes in the healthy controls $(\mathrm{HC})$, patients with Graves' disease, and children with type 1 diabetes mellitus (TIDM). Expression of the surface markers (A) CDI lb, (B) CDI lc, (C) CD62L, (D) CD68, and (E) CD163 on monocytes. Each dot represents a different individual; ns, $\mathrm{p}>0.05 ; *, \mathrm{p}<0.05 ; * *, \mathrm{p}<0.01 ; * * *, \mathrm{p}<0.001$. 


\section{Antigen-presenting and proinflammatory function of the intermediate monocytes in children with TIDM}

Because the changes of intermediate phenotypes were associated with the function of intermediate monocytes, we evaluated changes in the antigen-presenting function of this cell subset. The intermediate monocytes express high levels of MHC class II processing and presentation genes, and have been linked to antigen presentation in rheumatoid arthritis [30]. Therefore, we assessed the levels of molecules related to antigen presentation and co-stimulation on the intermediate monocytes of the children with T1DM.

HLA-DR expression suggests the antigen processing and presentation capability of the expanded subset [31]. The intermediate monocytes in T1DM patients were found to have significantly increased HLA-DR expression compared with those of the patients with Graves or the HC group (Fig. 3A). CD86 molecules are up-regulated in resting monocytes after induction by the CD40-CD40L interaction [32]. Therefore, we assessed the levels of molecules related to such co-stimulation (CD86) in the intermediate monocytes. The intermediate subset from patients with T1DM was also found to express significantly higher level of CD86 than those of the patients with Graves or the HC group, indicating the high antigen-presenting capability of this subset in
A

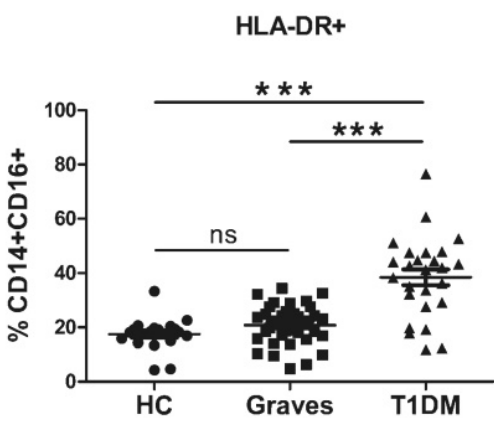

C

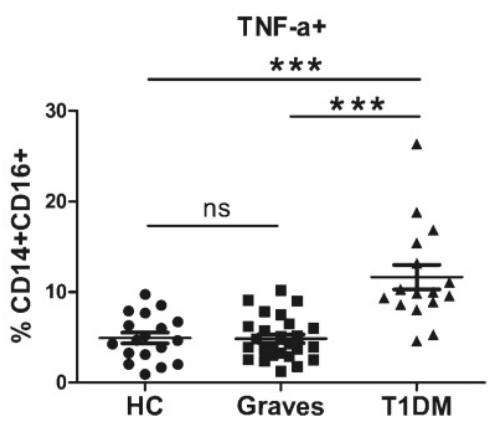

B

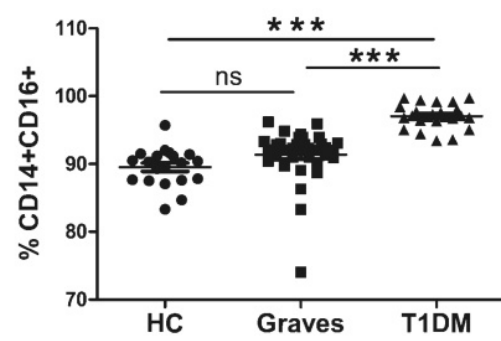

D

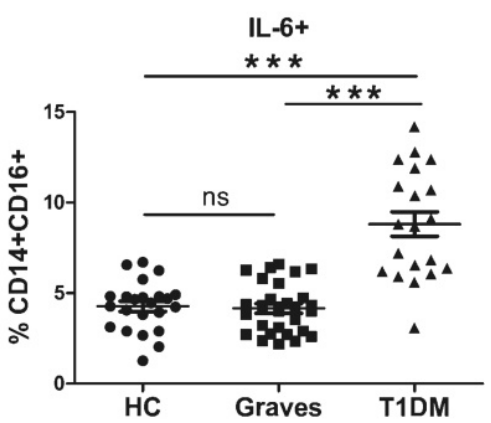

CD86+
Figure 3. The antigen-presenting function of CD14+CD16+ monocytes. (A) Expression of the surface markers (A) HLA-DR and (B) CD86 on monocytes. Levels of the cytokines (C) TNF- $\alpha$ and (D) IL-6 secreted by CD14+CD16+ monocytes. Each dot represents a different individual; ns, $\mathrm{p}>0.05 ; *, \mathrm{p}<0.05 ; * *, \mathrm{p}<0.01$; $* * *, \mathrm{p}<0.001$.

T1DM (Fig. 3B).

LPS priming could prevent diminished T-cell IFN- $\gamma$ production but had little effect on T-cell proliferation. Cros et al. [33] found that isolated human intermediate subset cells treated with LPS produced higher levels of TNF- $\alpha$, IL-1 $\beta$, and IL-6. Similarly, in our study, we detected strikingly increased production of TNF- $\alpha$ by the circulating intermediate monocytes from the T1DM subjects (Fig. $3 \mathrm{C})$, suggesting a systemic alteration in the function of a subset of monocytes/macrophages. Intermediate monocytes derived from the recent-onset T1DM subjects were found to have specifically increased numbers of TNF- $\alpha$-secreting cells compared with those derived from patients with Graves or the HC group. Interestingly, we also observed an increased number of IL-6-secreting cells in the intermediate monocytes from patients with T1DM compared with those of patients with Graves or HC (Fig. 3D).

\section{Relationship between monocytes and memory T cells in children with TIDM}

Monocytes may promote the expansion of memory $\mathrm{T}$ cells in patients with HIV [25]. Furthermore, respiratory monocytes enhance the responses of $\mathrm{CD} 4+$ memory $\mathrm{T}$ cells to mucosal immunization with recombinant adenovirus-based vaccines [26]. Therefore, we tested whether memory $\mathrm{T}$ cells were increased in T1DM. As shown in Fig. 4A and $4 \mathrm{~B}$, the percentage and absolute count of memory

$\mathrm{T}$ cells were higher in the T1DM group than in the Graves and HC groups. We further investigated the relationship between the absolute numbers of intermediate and memory T cells. As shown in Fig. 4C, T1DM patients with higher numbers of intermediate cells also had a greater number of memory $\mathrm{T}$ cells. Our data demonstrated that there is no correlation between classical (or non-classical) and memory $\mathrm{T}$ cells in T1DM (Supplementary Figure $1 \mathrm{~B}, 1 \mathrm{C})$. This phenomenon is specific exist in intermediate and memory $\mathrm{T}$ cells. And we also analyzed the correlation between intermediate and memory $\mathrm{T}$ cells in $\mathrm{HC}$ and Graves (Supplementary Figure 2D, Supplementary Figure 3E), and found this correlation exists only in T1DM. 
Furthermore, CD40 expressed on monocytes provides the co-stimulatory signals necessary for $\mathrm{T}$ cell activation and survival [34]. CD40L is a cell-surface interaction molecule that is expressed by $\mathrm{T}$ cells, and its interaction with CD40 plays a key role in the adaptive immune response [35]. Our data demonstrated that intermediate monocytes of patients with T1DM expressed higher levels of CD40 than

A

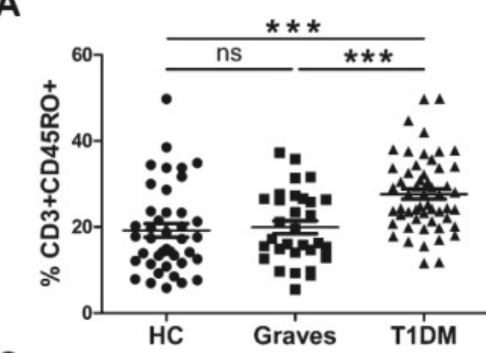

C

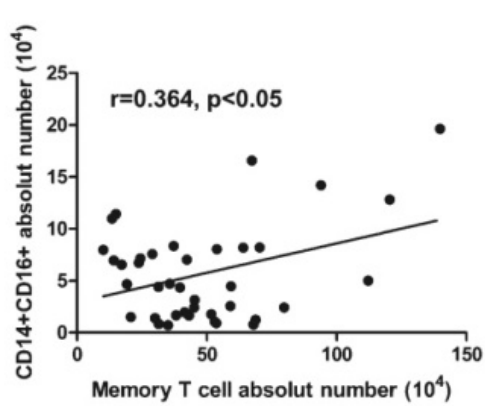

E

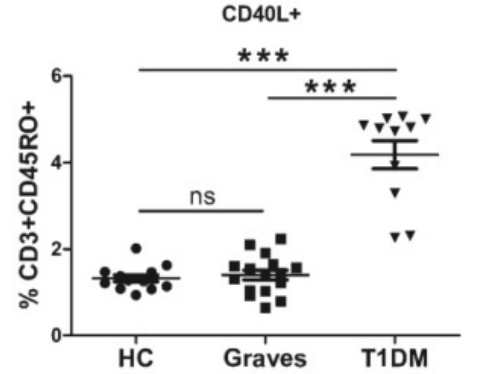

G

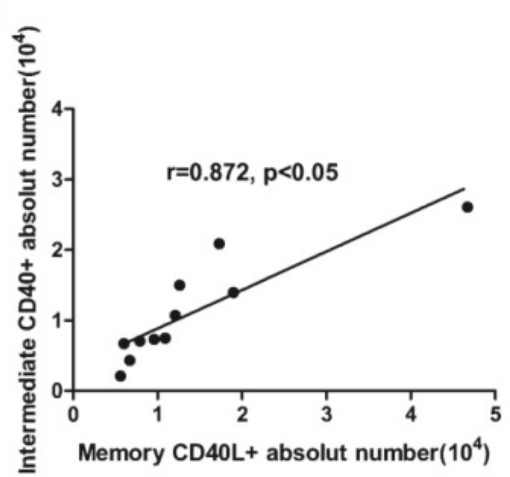

Figure 4. (A) Proportion (\%) of memory T cells among blood lymphocytes. (B) Absolute number of memory T cells. (C) The correlation of the absolute number of memory $T$ cells with the absolute number of CDI4+CD16+ monocytes ( $r=0.364, p<0.05)$. (D) Expression of CD40 in CD14+CD16+ monocytes. (E) Expression of CD40L in memory T cells. Each dot represents a different individual; $n s, p>0.05$; $(F)$ The correlation of the percentage of CD40L+ memory $T$ cells with the percentage of CD40+CD14+CD16+ monocytes $(r=0.701, p<0.05)$; $(G)$ The correlation of the absolute number of $\mathrm{CD} 40 \mathrm{~L}+$ memory $\mathrm{T}$ cells with the absolute number of CD40+CD 14+CD16+ monocytes $(r=0.872, \mathrm{p}<$ $0.05) ; *$, $<0.05 ; * *, p<0.01 ; * * *, p<0.001$. those of patients with Graves or the HC group (Fig. 4D). Moreover, compared with the HC or Graves patients, a significantly higher percentage of memory $\mathrm{T}$ cells from children with T1DM exhibited CD40L expression (Fig. 4E). Our data showed that T1DM patients with higher percentage of $\mathrm{CD} 40+$ in intermediate cells also had a greater percentage of $\mathrm{CD} 40 \mathrm{~L}+$ in memory $\mathrm{T}$ cells. This correlation remains
B
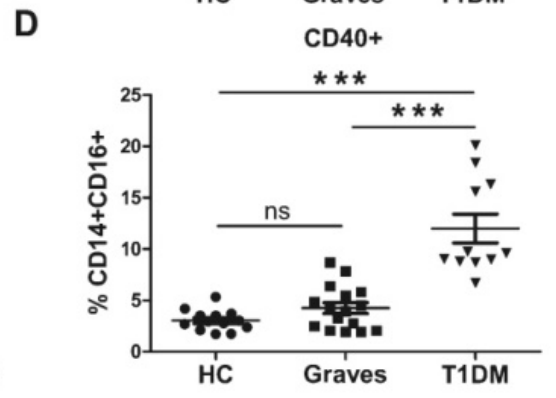

$\mathbf{F}$

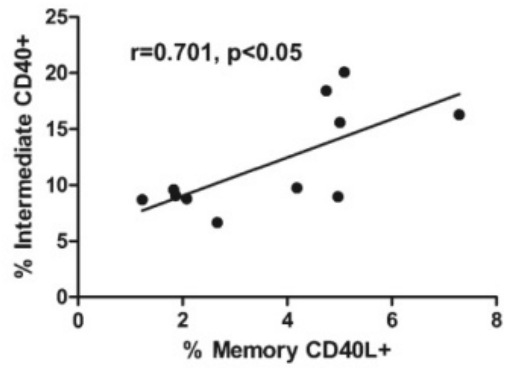
unchanged when absolute number of cells was considered (Fig. 4F and $4 G)$. This relationship does not exist in $\mathrm{HC}$ or Graves (Supplementary Figure 3A, 3B, 3C, 3D).

\section{Effector and central memory $T$ cells in children with TIDM}

To specifically evaluate which type of memory $\mathrm{T}$ cells increased in T1DM, we used the expression of CCR7 to separate out the memory $\mathrm{T}$ cells. We demonstrated that the proportion of CCR7+CD45RO+ central memory CD4+ $\mathrm{T}$ cells from patients with T1DM was increased as compared to that of patients with Graves and the $\mathrm{HC}$, respectively (Fig. 5A). Central memory $\mathrm{T}$ cells secrete the proinflammatory cytokine IL-2. IL-2 secretion analysis showed that the central memory $\mathrm{T}$ cells from patients with T1DM expressed higher levels of IL-2 (Fig. 5C). Furthermore, a higher frequency of CD4+ $\mathrm{T}$ cells from patients with T1DM presented a CCR7-CD45RO+ effector memory phenotype than those of patients with Graves and the HC (Fig. 5B). Moreover, the effector memory cells from patients with T1DM expressed the major effector cytokine IFN- $\gamma$ (Fig. 5D).

\section{Discussion}

Through investigation of 54 recent-onset T1DM children aged from 1 to 11 years, we found that intermediate monocytes were increased compared with the healthy cohort. This finding is in line with previous reports showing an expansion of the intermediate monocyte population and a reduction of classical monocytes in juvenile T1DM patients whose disease duration was 5 years [6]. This 
is also consistent with data showing that the CD16+ subset of monocytes is expanded in some autoimmune diseases and may be involved in the induction of the inflammatory immune response [36-38].

Recent gene profiling analyses suggest that the intermediate subset plays a critical role in antigen presentation $[39,40]$. A series of studies showed that the antigen presentation ability is tightly associated with HLA-DR and CD86 expression, suggesting an important role of HLA-DR and CD86 in the antigen presentation process $[31,32]$. We also demonstrated that the expression levels of CD86 and HLA-DR were increased in the intermediate monocytes of T1DM patients. Monocytes are producers of proinflammatory cytokines, including TNF- $\alpha$, IL-1, IL-6, and IL-12 [33, 39, 41, 42]. In our study, the intermediate monocytes of children with T1DM were found to secrete higher levels of IL- 6 and TNF- $\alpha$ than those of the HC group. Previous studies have shown that a higher $\mathrm{HbA} 1 \mathrm{c}$ level was associated with higher production of TNF- $\alpha$ [43], and that monocytes mediated the endothelial damage in chronic kidney disease patients by secreting cytokines [44]. In our study, an increased level of HbA1c correlated well with the increased absolute number of the intermediate subset. The levels of insulin and C-peptide are reliable clinical indicators for the
A

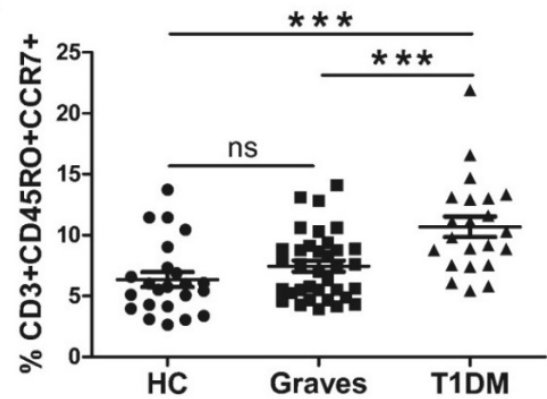

C

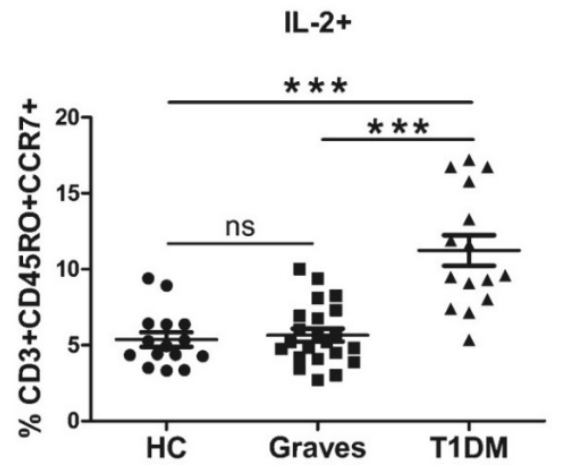

B

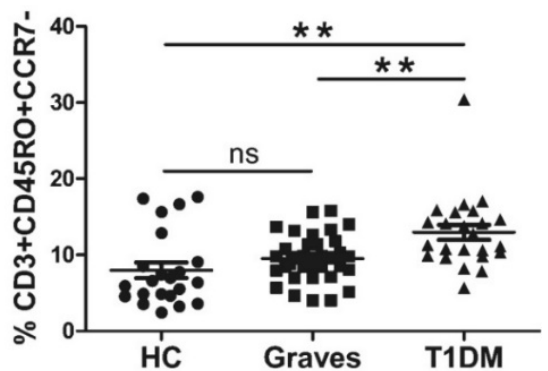

D

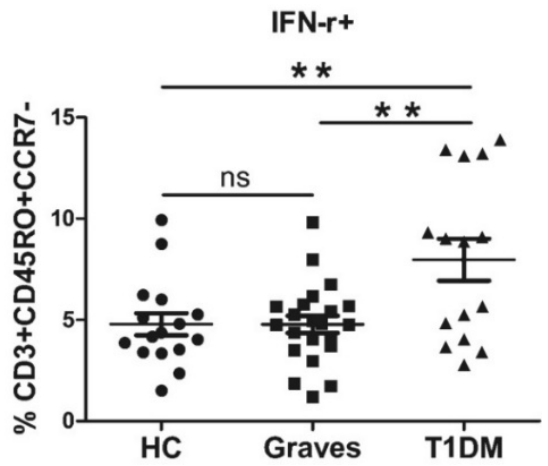

Figure 5. (A) Proportion (\%) of central memory T cells. (B) Proportion (\%) of effector memory T cells. (C) Level of IL-2 secreted by central memory T cells. (D) Level of IFN- $\gamma$ secreted by effector memory $T$ cells. Each dot represents a different individual; $n s, \mathrm{p}>0.05$ * $^{*}, \mathrm{p}<0.05$; **, $\mathrm{p}<0.01$; ***, $\mathrm{p}<0.001$. competent function of $\beta$-cell [45]. Our data demonstrated that the absolute number of intermediate monocytes was negatively correlated with the concentrations of C-peptide and insulin. Collectively, these results suggest that expanded intermediate monocytes might play a detrimental role for $\beta$-cell function in children with T1DM.

It is possible that the expansion of intermediate monocytes would result in the release of more proinflammatory cytokines, which would eventually lead to a detrimental effect on $\beta$-cells. It has been documented that continuous autocrine TNF- $\alpha$ stimulation of CD14+ monocytes drives the cells to differentiate into dendritic cells, which in turn incessantly produce TNF- $\alpha$ and other inflammatory factors [6]. Studies have also shown that intermediate monocytes are able to induce $\mathrm{CD} 4^{+} \mathrm{T}$ cells to produce IFN- $\gamma$, TNF- $\alpha$, and IL-17 in other autoimmune diseases $[6,46]$.

Based on our results and previous reports, it seems likely that intermediate CD14+CD16+ monocytes, which produce abundant TNF- $\alpha$, may play an important role in promoting the inflammatory response in T1DM [10]. Moreover, some authors have demonstrated that TNF- $\alpha$ provides a unique vantage point from which to characterize the cellular niches maintaining memory $\mathrm{T}$ cells [47]. Therefore, these correlations suggest that intermediate monocytes and memory $\mathrm{T}$ cells have a relationship to a certain degree. Given this background, we evaluated the correlation of the absolute numbers of memory $\mathrm{T}$ cells and intermediate monocytes in patients with T1DM, and also found a strong positive association between the two subsets in the peripheral blood. We further found that the percentage of proinflammatory memory $\mathrm{T}$ cells was expanded in children with T1DM. Moreover, the CD40-CD40L interaction has been proposed to play an essential role in mediating the interaction between $\mathrm{T}$ cells and APCs [48, 49]. We also found that CD40 and CD40L were increased in the intermediate monocytes and memory $\mathrm{T}$ cells of children 
with T1DM, respectively. And both of them were positively correlated. Our data demonstrated that the correlation between intermediate and memory $\mathrm{T}$ cells is specific in T1DM. Moreover, these cells secreted high levels of IL- 2 and IFN- $\gamma$. Thus, our hypothesis is that intermediate monocytes can promote the progress of T1DM in children and may be an indicator of pre-clinical T1DM.

In conclusion, the intermediate monocyte population was found to be expanded in pediatric patients with T1DM. As these cells were shown to have proinflammatory activity, they are likely to be implicated in the impaired function of $\beta$-cells, with deleterious consequences for the development of T1DM. Further characterization of the function of intermediate monocytes could pave the road for improving understanding of the relationships between these proinflammatory cells and the destruction of $\beta$-cells in children with T1DM.

\section{Supplementary Material}

Supplementary figures.

http://www.ijbs.com/v13p0209s1.pdf

\section{Acknowledgement}

This work was supported by a startup research fund to JG from Beijing Children's Hospital, a teaching hospital affiliated to Capital Medical University. This work was also partially supported by the National Natural Science Foundation of China (31470862) awarded to XN.

\section{Author Contributions}

Conceived and designed the experiments: JG, $\mathrm{CG}, \mathrm{XN}$; performed the experiments: $\mathrm{XR}, \mathrm{WM}, \mathrm{CS}$, $\mathrm{XC}, \mathrm{HZ}, \mathrm{BC}, \mathrm{XL}$; analyzed the data: $\mathrm{XR}, \mathrm{WM}$; contributed reagents/materials/analysis tools: JG, $\mathrm{XN}, \mathrm{CG}$, and DW; wrote the paper: XR, WM, JG, CG, CS.

\section{Competing Interests}

The authors have declared that no competing interest exists.

\section{References}

1. Ismail NA, Abd El Baky AN, Ragab S, Hamed M, Hashish MA, Shehata A. Monocyte chemoattractant protein 1 and macrophage migration inhibitory factor in children with type 1 diabetes. Journal of pediatric endocrinology \& metabolism : JPEM. 2016; 29: 641-5.

2. Xiao F, Ma L, Zhao M, Huang G, Mirenda V, Dorling A, et al. Ex vivo expanded human regulatory $\mathrm{T}$ cells delay islet allograft rejection via inhibiting islet-derived monocyte chemoattractant protein-1 production in $\mathrm{CD} 34+$ stem cells-reconstituted NOD-scid IL2rgammanull mice. PloS one. 2014; 9: e90387.

3. Jailwala P, Waukau J, Glisic S, Jana S, Ehlenbach S, Hessner M, et al. Apoptosis of CD4+ CD25(high) T cells in type 1 diabetes may be partially mediated by IL-2 deprivation. PloS one. 2009; 4: e6527.

4. Morgan NG, Leete P, Foulis AK, Richardson SJ. Islet inflammation in human type 1 diabetes mellitus. IUBMB life. 2014; 66: 723-34
5. Cernea S, Herold KC. Monitoring of antigen-specific CD8 T cells in patients with type 1 diabetes treated with antiCD3 monoclonal antibodies. Clinical immunology. 2010; 134: 121-9.

6. Mysliwska J, Smardzewski M, Marek-Trzonkowska N, Mysliwiec M, Raczynska K. Expansion of CD14+CD16+ monocytes producing TNF-alpha in complication-free diabetes type 1 juvenile onset patients. Cytokine. 2012; 60: 309-17.

7. Evans HG, Gullick NJ, Kelly S, Pitzalis C, Lord GM, Kirkham BW, et al. In vivo activated monocytes from the site of inflammation in humans specifically promote Th17 responses. Proceedings of the National Academy of Sciences of the United States of America. 2009; 106: 6232-7.

8. Evans HG, Suddason T, Jackson I, Taams LS, Lord GM. Optimal induction of T helper 17 cells in humans requires $\mathrm{T}$ cell receptor ligation in the context of Toll-like receptor-activated monocytes. Proceedings of the National Academy of Sciences of the United States of America. 2007; 104: 17034-9.

9. Acosta-Rodriguez EV, Rivino L, Geginat J, Jarrossay D, Gattorno M, Lanzavecchia A, et al. Surface phenotype and antigenic specificity of human interleukin 17-producing T helper memory cells. Nature immunology. 2007; 8: 639-46.

10. Ryba-Stanislawowska M, Mysliwska J, Juhas U, Mysliwiec M. Elevated levels of peripheral blood CD14(bright) CD16+ and CD14(dim) CD16+ monocytes may contribute to the development of retinopathy in patients with juvenile onset type 1 diabetes. APMIS : acta pathologica, microbiologica, et immunologica Scandinavica. 2015; 123: 793-9.

11. Ziegler-Heitbrock L, Ancuta P, Crowe S, Dalod M, Grau V, Hart DN, et al. Nomenclature of monocytes and dendritic cells in blood. Blood. 2010; 116: e74-80.

12. Zawada AM, Rogacev KS, Rotter B, Winter P, Marell RR, Fliser D, et al. SuperSAGE evidence for CD14++CD16+ monocytes as a third monocyte subset. Blood. 2011; 118: e50-61.

13. Ancuta P, Weiss L, Haeffner-Cavaillon N. CD14+CD16++ cells derived in vitro from peripheral blood monocytes exhibit phenotypic and functional dendritic cell-like characteristics. European journal of immunology. 2000; 30: 1872-83.

14. Wong KL, Tai JJ, Wong WC, Han H, Sem X, Yeap WH, et al. Gene expression profiling reveals the defining features of the classical, intermediate, and nonclassical human monocyte subsets. Blood. 2011; 118: e16-31.

15. Palmer CS, Anzinger JJ, Zhou J, Gouillou M, Landay A, Jaworowski A, et al. Glucose transporter 1-expressing proinflammatory monocytes are elevated in combination antiretroviral therapy-treated and untreated HIV+ subjects. Journal of immunology. 2014; 193: 5595-603.

16. Heine GH, Ortiz A, Massy ZA, Lindholm B, Wiecek A, Martinez-Castelao A, et al. Monocyte subpopulations and cardiovascular risk in chronic kidney disease. Nature reviews Nephrology. 2012; 8: 362-9.

17. Zawada AM, Rogacev KS, Schirmer SH, Sester M, Bohm M, Fliser D, et al. Monocyte heterogeneity in human cardiovascular disease. Immunobiology. 2012; 217: 1273-84

18. Terasawa T, Aso Y, Omori K, Fukushima M, Momobayashi A, Inukai T. Bezafibrate, a peroxisome proliferator-activated receptor alpha agonist, decreases circulating $\mathrm{CD} 14(+) \mathrm{CD} 16(+)$ monocytes in patients with type 2 diabetes. Translational research : the journal of laboratory and clinical medicine. 2015; 165: 336-45.

19. Hepburn AL, Mason JC, Davies KA. Expression of Fcgamma and complement receptors on peripheral blood monocytes in systemic lupus erythematosus and rheumatoid arthritis. Rheumatology. 2004; 43: 547-54.

20. Saha P, Geissmann F. Toward a functional characterization of blood monocytes. Immunology and cell biology. 2011; 89: 2-4.

21. Joussen AM, Doehmen S, Le ML, Koizumi K, Radetzky S, Krohne TU, et al. TNF-alpha mediated apoptosis plays an important role in the development of early diabetic retinopathy and long-term histopathological alterations. Molecular vision. 2009; 15: 1418-28.

22. Zorena K, Mysliwska J, Mysliwiec M, Balcerska A, Lipowski P, Raczynska K. Interleukin-12 and tumour necrosis factor-alpha equilibrium is a prerequisite for clinical course free from late complications in children with type 1 diabetes mellitus. Scandinavian journal of immunology. 2008; 67: 204-8.

23. Zorena K, Mysliwska J, Mysliwiec M, Balcerska A, Hak L, Lipowski P, et al. Serum TNF-alpha level predicts nonproliferative diabetic retinopathy in children. Mediators of inflammation. 2007; 2007: 92196.

24. Bradshaw EM, Raddassi K, Elyaman W, Orban T, Gottlieb PA, Kent SC, et al. Monocytes from patients with type 1 diabetes spontaneously secrete proinflammatory cytokines inducing Th17 cells. Journal of immunology. 2009; 183: 4432-9.

25. Judge CJ, Sandberg JK, Funderburg NT, Sherman KE, Butt AA, Kang M, et al. CD14brightCD16- monocytes and sCD14 level negatively associate with CD4-memory T-cell frequency and predict HCV-decline on therapy. Journal of acquired immune deficiency syndromes. 2016.

26. Acosta-Ramirez E, Tram C, Kampen RM, Tillman MR, Schwendener RA, Xing $\mathrm{Z}$, et al. Respiratory macrophages regulate CD4 $\mathrm{T}$ memory responses to mucosal immunization with recombinant adenovirus-based vaccines. Cellular immunology. 2016.

27. Rodriguez-Garcia M, Shen Z, Barr FD, Boesch AW, Ackerman ME, Kappes JC, et al. Dendritic cells from the human female reproductive tract rapidly capture and respond to HIV. Mucosal immunology. 2016.

28. Wallquist C, Mansouri L, Norrback M, Hylander B, Jacobson SH, Lundahl J. Early Changes in Monocyte Adhesion Molecule Expression and Tumor 
Necrosis Factor-alpha Levels in Chronic Kidney Disease - A 5-Year Prospective Study. American journal of nephrology. 2016; 44: 268-75.

29. Raggi C, Correnti M, Sica A, Andersen JB, Cardinale V, Alvaro D, et al. Cholangiocarcinoma Stem-like Cells Shapes Tumor-initiating Niche by Regulating Associated Macrophages. Journal of hepatology. 2016.

30. Nagafuchi Y, Shoda H, Sumitomo S, Nakachi S, Kato R, Tsuchida Y, et al. Immunophenotyping of rheumatoid arthritis reveals a linkage between HLA-DRB1 genotype, CXCR4 expression on memory CD4(+) T cells, and disease activity. Scientific reports. 2016; 6: 29338.

31. Wang D, Yuan F, Wang L, Wei W. Paeoniflorin inhibits function and down-regulates HLA-DR and CD80 expression of human peripheral blood monocytes stimulated by rhIL-1beta. International immunopharmacology. 2012; 14: 172-8.

32. Beyranvand Nejad E, van der Sluis TC, van Duikeren S, Yagita H, Janssen GM, van Veelen PA, et al. Tumor eradication by cisplatin is sustained by CD80/86-mediated costimulation of CD8+ T cells. Cancer research. 2016.

33. Cros J, Cagnard N, Woollard K, Patey N, Zhang SY, Senechal B, et al. Human CD14dim monocytes patrol and sense nucleic acids and viruses via TLR7 and TLR8 receptors. Immunity. 2010; 33: 375-86.

34. Samitas K, Malmhall C, Radinger M, Ramos-Ramirez P, Lu Y, Deak T, et al. Precursor B Cells Increase in the Lung during Airway Allergic Inflammation: A Role for B Cell-Activating Factor. PloS one. 2016; 11: e0161161.

35. Azzariti A, Brunetti O, Porcelli L, Graziano G, Iacobazzi RM, Signorile M, et al. Potential predictive role of chemotherapy-induced changes of soluble CD40 ligand in untreated advanced pancreatic ductal adenocarcinoma. OncoTargets and therapy. 2016; 9: 4681-6.

36. Kawanaka N, Yamamura M, Aita T, Morita Y, Okamoto A, Kawashima M, et al. CD14+,CD16+ blood monocytes and joint inflammation in rheumatoid arthritis. Arthritis and rheumatism. 2002; 46: 2578-86.

37. Ramirez R, Carracedo J, Merino A, Soriano S, Ojeda R, Alvarez-Lara MA, et al. CD14+CD16+ monocytes from chronic kidney disease patients exhibit increased adhesion ability to endothelial cells. Contributions to nephrology. 2011; 171: 57-61.

38. Rossol M, Kraus S, Pierer M, Baerwald C, Wagner U. The CD14(bright) CD16+ monocyte subset is expanded in rheumatoid arthritis and promotes expansion of the Th17 cell population. Arthritis and rheumatism. 2012; 64: 671-7.

39. Mitchell A, Rentero C, Endoh Y, Hsu K, Gaus K, Geczy C, et al. LILRA5 is expressed by synovial tissue macrophages in rheumatoid arthritis, selectively induces pro-inflammatory cytokines and IL-10 and is regulated by TNF-alpha, IL-10 and IFN-gamma. European journal of immunology. 2008; 38: 3459-73.

40. Hristov $\mathrm{M}$, Weber $\mathrm{C}$. Differential role of monocyte subsets in atherosclerosis. Thrombosis and haemostasis. 2011; 106: 757-62.

41. Devaraj S, Dasu MR, Rockwood J, Winter W, Griffen SC, Jialal I. Increased toll-like receptor (TLR) 2 and TLR4 expression in monocytes from patients with type 1 diabetes: further evidence of a proinflammatory state. The Journal of clinical endocrinology and metabolism. 2008; 93: 578-83.

42. Ancuta P, Rao R, Moses A, Mehle A, Shaw SK, Luscinskas FW, et al. Fractalkine preferentially mediates arrest and migration of CD16+ monocytes. The Journal of experimental medicine. 2003; 197: 1701-7.

43. Aiello LP, Wong JS. Role of vascular endothelial growth factor in diabetic vascular complications. Kidney international Supplement. 2000; 77: S113-9.

44. Merino A, Portoles J, Selgas R, Ojeda R, Buendia P, Ocana J, et al. Effect of different dialysis modalities on microinflammatory status and endothelial damage. Clinical journal of the American Society of Nephrology : CJASN. 2010; 5: 227-34.

45. Dereke J, Nilsson C, Strevens H, Landin-Olsson M, Hillman M. IgG4 subclass glutamic acid decarboxylase antibodies (GADA) are associated with a reduced risk of developing type 1 diabetes as well as increased C-peptide levels in GADA positive gestational diabetes. Clinical immunology. 2016; 162: 45-8.

46. Liu B, Dhanda A, Hirani S, Williams EL, Sen HN, Martinez Estrada F, et al. CD14++CD16+ Monocytes Are Enriched by Glucocorticoid Treatment and Are Functionally Attenuated in Driving Effector T Cell Responses. Journal of immunology. 2015; 194: 5150-60.

47. Sabbagh L, Snell LM, Watts TH. TNF family ligands define niches for T cell memory. Trends in immunology. 2007; 28: 333-9.

48. Cabral-Marques O, Ramos RN, Schimke LF, Khan TA, Amaral EP, Barbosa Bomfim CC, et al. Human CD40L deficiency dysregulates the macrophage transcriptome causing functional defects that are improved by exogenous IFN-gamma. The Journal of allergy and clinical immunology. 2016.

49. Durlanik S, Loyal L, Stark R, Alp OS, Hartung A, Radbruch A, et al. CD40L expression by CD4+ but not CD8+ $\mathrm{T}$ cell regulates antiviral immune responses in acute LCMV infection in mice. European journal of immunology. 2016. 